\title{
Tests of a holistic chunking model of sentence memory through analyses of noun intrusions
}

\author{
ARTHUR C. GRAESSER \\ California State University, Fullerton, California 92634
}

\begin{abstract}
A holistic chunking model of sentence acquisition and retrieval is described and tested by a prompted sentence recall procedure. In this procedure, subjects first study a list of unrelated sentences and later receive single-word prompts to cue sentence recall. The model assumes that (1) words in sentences are grouped into propositions during acquisition, (2) the propositions are encoded holistically and later retrieved as units, and (3) the retrieval of one proposition does not automatically lead to recovery of other propositions in a sentence. The model was tested by patterns of intrusion errors. Noun intrusions for elements within a recovered proposition were always related conceptually to the presented nouns, even when a noun violated the co-occurrence restrictions of the verb (e.g., the tray loved the house). In contrast, noun intrusions for elements outside of the scope of a recovered proposition were often unrelated to presented nouns. It was argued that patterns of intrusion errors provide more appropriate tests for sentence structure than do quantitative patterns of correct recall, at least from the framework of the holistic chunking model.
\end{abstract}

The acquisition and recall of sentences has been a popular paradigm for examining the representation of verbal material. In most studies, the sentences are simple and are not embedded in a referential or discourse context. Two questions will be pursued in this study: How are words in sentences grouped into constituents? Are any of the constituents encoded in a holistic unitary fashion? A holistic chunking model of sentence acquisition and recall will be pursued in the course of examining these questions. In addition, the present study will segregate some measures of memory performance that provide adequate tests of the holistic chunking model. Recent reports have critically reexamined the role of behavioral data in testing theories of representation (J. R. Anderson, 1976; J. R. Anderson \& Bower, 1973; Kosslyn \& Pomeranz, 1977; Norman \& Bobrow, 1975).

\section{A HOLISTIC CHUNKING MODEL OF SENTENCE ENCODING AND RETRIEVAL}

A holistic chunking model is proposed to account for the acquisition and retrieval of unrelated sentences in memory experiments. The four assumptions below

Experiment 1 was supported by National Science Foundation Grant GB20798 and served as partial fulfillment of a doctoral dissertation. The author would like to thank the members of his dissertation committee, George Mandler (advisor), David E. Rumelhart, Lisa L. Newport, Roy G. D'Andrade, and Tim S. Smith, for their helpful advice and support. Wayne J. Boeck, Cheryl C. Graesser, Allen Munro, Donald A. Norman, Jan C. Rabinowitz, and Stanley Woll also provided constructive comments on earlier drafts of the manuscript. Requests for reprints should be sent to Arthur C. Graesser, Department of Psychology, California State University, Fullerton, California 92634. directly address the issues of segmentation and holism. Assumption 1. Some sentence constituents are encoded holistically.

Assumption 2. Sentence "predicates" (verbs, adjectives, prepositions, and connectives) are important determinants of the segmentation of words into constituents, and how the constituents are organized structurally.

Assumption 3. Sentences are segmented into propositions, which contain a predicate and one or more arguments. Propositions are encoded and retrieved as holistic units.

Assumption 4. The retrieval of one proposition in a sentence does not insure retrieval of other propositions.

Assumption 1 has received support from several studies in memory and psycholinguistics (R. C. Anderson \& Ortony, 1975; Fodor, Bever, \& Garrett, 1974; Foss \& Harwood, 1973; Green, 1973; Jenkins, 1974; Marschark \& Paivio, 1977). The interpretation, utilization, and retrieval of holistic constituents are believed to operate in a unitary fashion. The retrieval of a holistic unit is said to be redintegrative (Asch, 1969; Greeno, 1970; Hayes-Roth, 1977; Horowitz \& Prytulak, 1969; Johnson, 1972), which means that the memory activation of one element in a holistic constituent will ultimately reinstate fragments of the entire constituent. Not all models of sentence acquisition and retrieval have assumed the existence of holistic units (J. R. Anderson, 1976; J. R. Anderson \& Bower, 1973; Thorndyke, 1975). For example, the existence of holistic encodings was directly rejected in J. R. Anderson and Bower's (1973) HAM model of sentence acquisition and memory.

Assumption 2 is consistent with a number of theories of representation (Clark \& Clark, 1977; Fillmore, 1968; 
Fodor et al., 1974; Kintsch, 1974; Norman, Rumelhart, \& The LNR Research Group, 1975; Rumelhart \& Ortony, 1976; Simmons, 1973). Predicates direct the course of segmenting sentences into units called propositions. A proposition contains a predicate and one or more arguments. The predicate of "John hit Mary" is "hit," whereas "John" and "Mary" are the arguments. Predicates also determine how the propositions in a sentence are structurally related. The sentence "the gambler borrowed the money in the park" has two propositions: (1) PROP1 (borrow, gambler, money) and (2) PROP2 (in, PROP1, park). PROP1 is embedded in PROP2. Assumption 3 states that propositions are the functional holistic units, whereas Assumption 4 states that the retrieval of one proposition in a sentence will not always provide access to other sentence propositions.

\section{METHODS OF TESTING THE HOLISTIC CHUNKING MODEL}

A popular procedure for examining the memory representations of sentences is the prompted sentence recall paradigm (J. R. Anderson \& Bower, 1971, 1973; Blumenthal, 1967; Blumenthal \& Boakes, 1967; Foss \& Harwood, 1975; Wanner, 1968). During the acquisition phase, subjects study a list of unrelated sentences; at the test phase, the subjects receive one or more words as prompts to cue recall for the sentences in which the prompt words occurred. The experiment in this study involves single-word prompts. For example, the test word "gambler" is presented to prompt recall for the acquisition sentence "the gambler borrowed the money in the park." Over the entire experiment, prompt words may come from any of the four main sentence positions: subject $(\mathrm{S})$, verb $(\mathrm{V})$, object $(\mathrm{O})$, and final argument $(\mathrm{A})$. The pattern of recall errors is expected to reflect the structure of the stored memory representations.

The process of recalling an acquisition sentence may conveniently be divided into three stages: (1) trace access, (2) trace decoding, and (3) word reconstruction. Trace access includes processes involved in recovering the appropriate sentence trace (memory representation) on the basis of the attributes that are activated from the prompt word. Word reconstruction refers to the process of reconstructing lexical items when a sentence trace has been recovered. Trace access and word reconstruction involve pattem-match processes between contextually specific traces and lexical representations (cf. Kintsch, 1974).

Trace decoding refers to the reinstatement of attributes, elements, and propositions of sentence traces whenever the sentence context is recovered. According to the holistic chunking model, there are two assertions about trace decoding. First, some attributes of each element within a particular proposition will be recovered whenever the proposition is accessed. Second, access to one proposition does not automatically lead to recovery of another proposition.
The three stages of sentence retrieval permit a mathematically tractable account of the prompted sentence recall paradigm. Let $\mathrm{Mn}$ refer to the memory trace for the main proposition, PROP1 $(\mathrm{S}, \mathrm{V}, \mathrm{O})$, and $\mathrm{Mf}$ refer to the modifier proposition, which includes some of the attributes of the main proposition, $\mathrm{Mn}^{\prime}$, and the final argument: PROP2 (in, $\mathrm{Mn}^{\prime}, \mathrm{A}$ ). The $\mathrm{Mn}^{\prime}$ argument of PROP2 is not necessarily equivalent to the Mn proposition, since modifiers qualify certain aspects of another proposition rather than all aspects. The process of trace decoding contains the following two parameters, which correspond to the likelihood that one proposition will recover another proposition: (1) $p(M n \rightarrow M f)=p$ and (2) $\mathrm{p}(\mathrm{Mf} \rightarrow \mathrm{Mn})=\mathrm{q}$. Trace access involves pattern-match processes between the prompt word and the sentence trace. There is a parameter for each sentence position, which corresponds to the likelihood that a prompt word will recover the sentence proposition with that prompt word: (3) $\mathrm{p}(\mathrm{S} \rightarrow \mathrm{Mn})=\mathrm{s}, \quad$ (4) $\mathrm{p}(\mathrm{V} \rightarrow \mathrm{Mn})=\mathrm{v}$, (5) $\mathrm{p}(\mathrm{O} \rightarrow \mathrm{Mn})=\mathrm{o}$, and $(6) \mathrm{p}(\mathrm{A} \rightarrow \mathrm{Mf})=\mathrm{a}$. Finally, the process of word reconstruction may be captured by four parameters, one for each content word in a sentence: (7) $\mathrm{p}(\mathrm{Mn} \rightarrow \mathrm{S})=\mathrm{S},(8) \mathrm{p}(\mathrm{Mn} \rightarrow \mathrm{V})=\mathrm{V},(9) \mathrm{p}(\mathrm{Mn} \rightarrow \mathrm{O})=0$, and $(10) \mathrm{p}(\mathrm{Mf} \rightarrow \mathrm{A})=\mathrm{A}$.

With the parameters listed in Equations $1-10$, it is possible to compute the likelihood that a particular prompt word will correctly recover a particular to-berecalled word. For example, the probability that a subject-noun prompt will access the correct verb is $\mathrm{s} \cdot \mathrm{V}$. The probability that a subject-noun prompt will recover the correct final argument is $s \cdot p \cdot A$.

Having described the holistic chunking model both conceptually and mathematically, it is possible to review some analyses that test whether it is plausible. There are three basic measures that have often been used to test hypothetical memory representations: (1) access probabilities, (2) conditional recall probabilities (CRPs), and (3) qualitative analyses of intrusion errors. Access probabilities measure the proportion of recall protocols in which a prompt word successfully recovers an acquisition sentence. CRPs come in many varieties, but generally they correspond to the proportion of recalls in which a particular to-be-recalled word is successfully retrieved, given that some other word or words are recovered. The qualitative analyses of intrusion errors will be discussed shortly.

The rationale behind the use of access probabilities and CRPs seems straightforward at first glance. For example, the holistic chunking model assumes that the verb is holistically tied to the subject and object nouns but not to the final argument nouns. It might be predicted, therefore, that the access probabilities and CRPs for A would be lower than those for $S$ and $O$. From the perspective of the holistic chunking model, however, access probabilities and CRPs do not provide defensible tests of the structure of memory representations. These measures would be satisfactory if there were some insurance that parameters $\mathrm{S}, \mathrm{O}$, and $\mathrm{A}$ are in fact 
equal, and that parameters $s, o$, and a are also equal. However, the three word-reconstruction parameters and the three access parameters may not be equal for two reasons. First, there may be an item-selection bias. The investigator might unintentionally assign different classes of nouns to the various arguments of acquisition sentences (Clark, 1965). Second, the subjects may differentially allocate their processing resources across arguments within a holistic unit during acquisition (Kahneman, 1973; Norman \& Bobrow, 1975). All of the arguments within a proposition may be integrated together holistically, but subjects may rehearse and form richer encodings for some of the arguments. Some elements may be more important than others when events are internally constructed (Haviland \& Clark, 1974; Singer, 1976).

Conditional omission errors, that is, omitting a response altogether when a sentence trace is accessed, do not provide an adequate test of the holistic chunking model, despite earlier claims that these omissions are inconsistent with a holistic framework. According to the holistic chunking model, omissions occur whenever a subject recovers a proposition from memory but is unable to generate a lexical item that adequately captures the configuration of attributes for an element.

Whereas there are severe problems with access probabilities and CRPs in testing the holistic chunking model, analyses of the intrusion errors may be more defensible. The holistic chunking model makes strong predictions about the intrusion errors for $\mathrm{S}, \mathrm{O}$, and $\mathrm{A}$ arguments. Whenever the main proposition is accessed, the intrusion errors for $\mathrm{S}$ and $\mathrm{O}$ arguments should always be conceptually related to the presented nouns, since the model claims there will always be some trace attributes available to direct word reconstruction. Moreover, the incidence of "related" intrusion errors for $\mathbf{S}$ and $\mathrm{O}$ arguments should not be sensitive to the amount of processing resources that were allocated to the arguments at input. In contrast, the intrusion errors for final argument nouns may not always be conceptually related to the presented nouns whenever (1) the main proposition is recovered, but not the modifier proposition [i.e., $\mathrm{p}(\mathrm{Mn} \rightarrow \mathrm{Mf})=0.0$ ], and (2) subjects guess a final argument. The predictions of the holistic chunking model can be tested by observing the conditionalized intrusion errors, that is, the intrusions that occur when a sentence trace is accessed.

A test of the holistic chunking model requires decisive criteria for segregating those intrusions that are related to the correct nouns from those that are unrelated. Previous studies have examined intrusions as tests for holistic encodings (R. C. Anderson, 1974; Brewer, 1975) but have not provided sufficiently decisive criteria for segregating related from unrelated intrusions. In these studies, errors were categorized into synonyms ("lady" for "woman"), superordinates ("lady" for "nurse"), subordinates ("nurse" for "lady"), and phonemically similar errors ("lad" for "lady"). Unfortunately, it is often difficult to defend some of these categories. Indeed, some theorists would claim that no two words are synonyms in a given language. The present decision criteria are based on the premise that certain semantic attributes of words are invariantly encoded at input, at least for the class of sentences that will be investigated. The invariantly encoded attributes correspond to some of the semantic features that were discussed in early linguistic theories of semantics (Chomsky, 1965; Katz \& Postal, 1964): \pm abstract, \pm animate, \pm human, and \pm male. The encoding of many of these semantic features is needed for performing syntactic analyses.

There are four categories of nouns that are mutually exclusive and sufficiently distinct for judges to agree on how to categorize nouns: abstract (rumor, problem), nonhuman concrete (box, ship), human male (man, father), and human female (girl, aunt). To accommodate all possible intrusions, there is a fifth category of human neutral sex nouns (baby, person). Whereas the first four categories are mutually exclusive, it is possible for the neutral sex nouns to be intrusions for male and female nouns, and vice versa. "Man" and "father" are unquestionably male, and "girl" and "princess" are undeniably female. However, there are nouns such as "secretary," "judge," and "minister" that by current cultural standards are usually, but not always, male or female. These normatively sex-specified nouns are included in the neutral sex category.

The proposed criterion of segregating unrelated and related intrusions includes both phonemic and semantic decisions. It is possible for an intrusion to violate the above five semantic categories and be obviously related both semantically and phonemically (e.g., "waitress" for "waiter," "bicycler" for "bicycle," etc.). An intrusion is scored as unrelated to the correct noun if it is both (1) not phonemically related and (2) a semantic violation. The intrusion matrix in Table 1 summarizes the criteria for segregating related and unrelated intrusions.

Table 1

Intrusion Matrix and Criteria for Segregating Related and Unrelated Intrusion Errors

\begin{tabular}{|c|c|c|c|c|c|c|}
\hline \multirow{3}{*}{$\begin{array}{c}\text { Presented } \\
\text { Noun } \\
\text { Category }\end{array}$} & \multicolumn{6}{|c|}{ Recalled Noun Category (Intrusions) } \\
\hline & \multirow{2}{*}{ Similar } & \multicolumn{4}{|c|}{ Dissimilar } & \multirow[b]{2}{*}{ NS } \\
\hline & & $\mathbf{A}$ & C & $\mathbf{M}$ & F & \\
\hline A & $*$ & * & & & & \\
\hline C & $*$ & & $*$ & & & \\
\hline $\mathbf{M}$ & $*$ & & & * & & $*$ \\
\hline $\mathbf{F}$ & $*$ & & & & * & * \\
\hline NS & * & & & * & $*$ & $*$ \\
\hline
\end{tabular}

Note-"Similar" refers to semantically unrelated but phonemically similar; "dissimilar" refers to phonemically dissimilar. $A=$ abstract,$C=$ concrete,$\quad M=$ male, $F=$ female, and $N S=$ neutral sex. "Intrusion errors in these cells are scored as related to the presented nouns. 
Experiment 1 was conducted to test the strong predictions of the holistic chunking model with regard to the intrusion errors. The conditionalized intrusion errors for subject and object nouns are predicted always to be related to the correct nouns, whereas the intrusions for final argument nouns are expected to be unrelated in some observations. Access probabilities and CRPs will be examined in addition to the qualitative analysis of intrusions. It is believed that the magnitudes of the CRPs and access probabilities primarily depend on the success of pattern-match processes during word reconstruction and trace access, as opposed to the structural properties of traces.

\section{EXPERIMENT 1}

\section{Method}

Subjects. Sixty undergraduates at the University of California, San Diego, participated to fulfill a requirement in an introductory psychology course.

Materials. Ninety-six sentences with a subject-verb-objectargument format were constructed with high- and moderately high-frequency words (Kučera \& Francis, 1967). The verbs were always presented in the past tense and the nouns were preceded by the article "the." The final arguments were in the form of a prepositional phrase. Sentences were constructed so that preexperimental associations among the words were minimal. Two sample sentences are "the daughter hated the success of the fellow" and "the bachelor punched the house despite the uncle."

The distribution of the five noun categories among the sentences was the same for subject and object positions: 16 abstract, 24 concrete, 14 male, 14 female, and 28 neutral sex. For reasons that will be explained shortly, the distribution of noun categories for the final argument was slightly different: 40 abstract, 18 concrete, 9 male, 9 female, and 20 neutral sex. Instances of the five categories were randomly assigned to the three sentence positions.

An appropriate selection of verbs was crucial for a meaningful analysis of the intrusions. The verbs did not have selectional restrictions that completely covaried with particular noun categories. Fortunately, there are a large number of verbs that can occur with any subject noun (frighten, worry) and other verbs that can occur with any object noun (hate, reject). For example, any entity or event can frighten someone (e.g., rumors, trees, uncles, ladies, and babies). The co-occurrence restrictions of the verbs occasionally constrain the categories of subject and object nouns, but not the argument nouns. Therefore, to equilibrate a base rate for guessing correct noun categories across the three argument positions, there was a different distribution of noun categories in the final argument position. A group of control subjects was pretested to insure that the guessing base rates were comparable for the three noun arguments. These subjects received the sentences with one of the nouns (or prepositional phrases) missing and guessed what the correct word was. The proportion of guesses that were scored as "related" was $.45, .47$, and .40 for subject, object, and argument nouns, respectively.

The 96 sentences were arranged into 12 booklets, with 8 sentences followed by eight prompt words. The sentences and prompts were shuffled by hand with the restriction that the first two prompt words never correspond to the last two sentences studied. There were four sets of booklets, each of which had a different prompt word for any given sentence, but overall, each set of booklets had subject, verb, object, and argument prompts. Fifteen subjects were assigned to each of the four sets of booklets; the booklets were randomly ordered for each subject.

Procedure. The subjects were tested in groups of three to six and randomly assigned one of four sets of booklets. For each of the 12 blocks, the subjects studied eight sentences one at a time, and then were provided eight prompt words, one at a time. The subjects were given $10 \mathrm{sec}$ to study each sentence and $25 \mathrm{sec}$ per sentence for written recall. A tape-recorded voice cued the subjects when to turn each page of the booklet. The subjects were instructed to write down verbatim as much of the sentence as they could remember.

\section{Results}

An analysis of the intrusion errors was the primary test of the holistic chunking model. The model predicts that subject- and object-noun intrusions should always be related to the correct nouns whenever a sentence trace is accessed, whereas the intrusions of the final argument were not expected always to be related to the presented nouns. A sentence trace was scored as accessed if at least one word in the sentence was correctly recalled via the prompt word. Altogether, there were 230,222 , and 211 of the conditionalized intrusions for nouns in the subject, object, and argument positions, respectively.

The analysis of intrusion errors clearly supported the holistic chunking model. Subjects practically never gave unrelated intrusions to nouns in the subject and object positions, whereas there was a relatively high rate of unrelated intrusions for nouns in the final argument position. The proportions of unrelated noun intrusions were $.017, .009$, and .195 for nouns in the subject, object, and argument positions, respectively. For purposes of statistical analyses, the frequencies of unrelated intrusions were scored for $\mathrm{S}, \mathrm{O}$, and $\mathrm{A}$ items. The number of unrelated intrusions per sentence significantly differed from 0.0 for final argument nouns $\left[\min \mathrm{F}^{\prime}(1,111)=35.3, \mathrm{p}<.05\right]$, but not for subject and object nouns. Table 2 shows the intrusion matrices for nouns in subject, object, and argument positions.

Further analyses of the subject- and object-noun intrusions revealed that .062 of the intrusions were phonemically similar to the correct nouns, while .191 were synonyms and .392 were superordinates or subordinates. Therefore, a large proportion of the intrusions were semantically related to the correct nouns, but difficult to classify according to the categorization schemes used by previous researchers (R. C. Anderson, 1974; Brewer, 1975).

Access probabilities and CRPs were also examined as measures of sentence structure. The access probability for a prompt word was computed as the proportion of observations in which the prompt word recovered at least one other word in the sentence. The mean access probabilities were not significantly different for subject, object, and argument nouns $(.51, .53$, and .49 , respectively). The CRP for a word was computed as the proportion of observations in which a word was recalled correctly, given that at least one other word in the 
Table 2

Intrusion Matrix for Subject, Object, and Argument Nouns

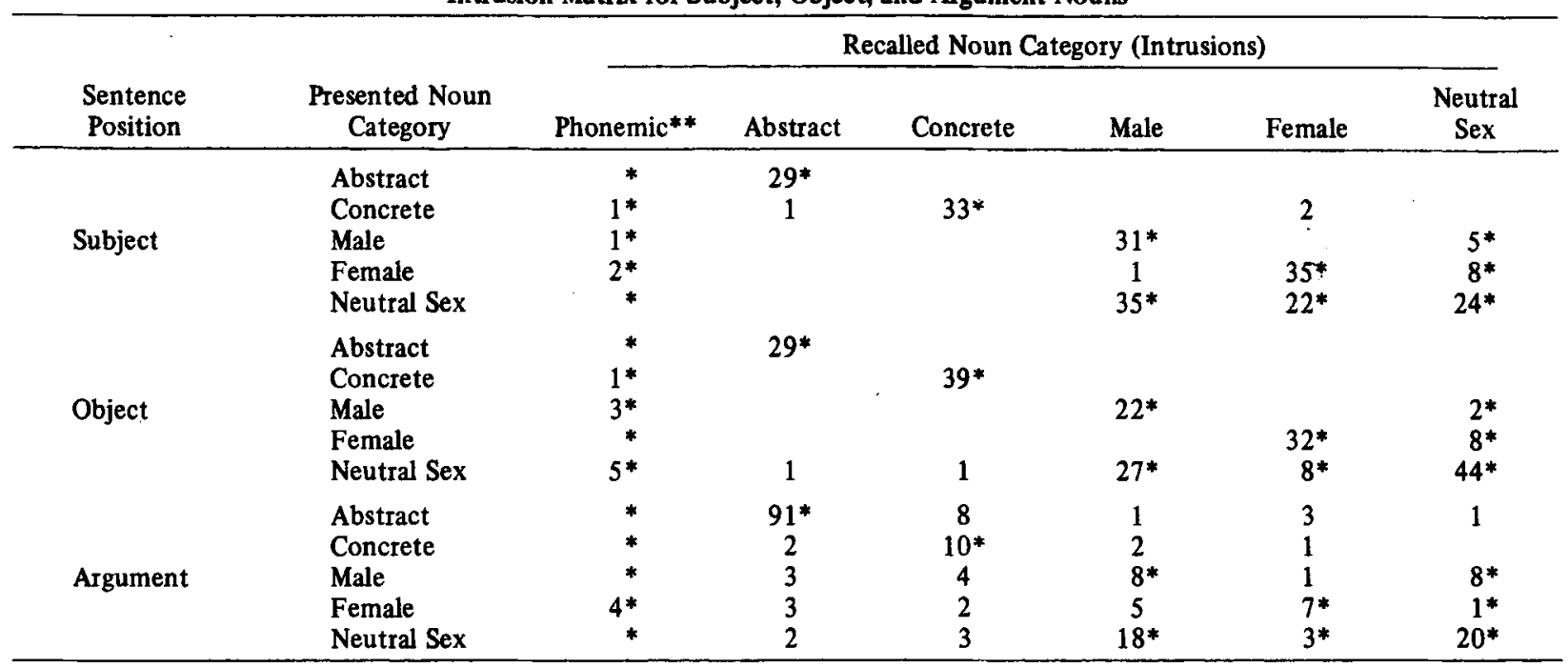

*Intrusions scored as related to the presented nouns.

**These intrusions were phonemically related to the presented nouns, but violated the semantic features.

sentence was correctly recalled. The mean CRPs were also not significantly different for subject, object, and argument nouns $(.75, .76$, and .73 , respectively). The access probabilities and CRPs therefore failed to expose the trace structure of sentences.

It was proposed earlier that the access probabilities and CRPs are good indices of the success of the patternmatch operations between sentence traces and lexical representations. Analyses were performed on the access probabilities and CRPs to examine the course of trace access and word reconstruction, respectively. Table 3 shows access probabilities and CRPs for the five noun categories: abstract, concrete, male, female, and neutral sex. Analyses focused on the subject and object nouns, since the final argument was not always integrated holistically with the SVO proposition. The magnitudes of the probabilities showed the same ordering for both access probabilities and CRPs: concrete $>$ male $>$ female $>$ neutral sex $>$ abstract. There were significant differences in mean access probabilities among the five noun categories $\left[\mathrm{min} \mathrm{F}^{\prime}(4,217)=6.83, \mathrm{p}<.05\right]$. It was not possible to perform the conservative min $F^{\prime}$ test (cf. Clark, 1973) on the CRPs because there were missing observations in some noun categories for some subjects. However, when an $F_{2}$ test was computed, using item variance as error, there were significant differences in mean CRPs among the five noun categories $\left[F_{2}(4,175)=5.61, p<.05\right]$. These data reveal that there are sizable variations among noun categories in the likelihood of successful pattern matches between sentence traces and lexical items.

A final set of analyses tested additional properties of pattern-match processes. The likelihood of a successful pattern match is expected to increase with the amount of overlap between the lexical attributes of the words and the attributes of elements in specific sentence traces (Kintsch, 1974). Moreover, the probability of a correct match should generally increase with the number of lexical attributes of the words (Tulving, 1976). When many attributes are encoded in a sentence trace, there are fewer alternative words that fit during word reconstruction. Similarly, when a prompt word contains many lexical attributes, there are fewer sentence traces that contain the feature pattern activated by the prompt word. The male and female nouns were analyzed to test this prediction.

A subset of the male and female nouns was divided into three "levels," varying from a general level with few features to a specific level with many features (cf. Miller, 1969). Level 1, the most general level, included nouns with a minimum of features, namely, sex and age (e.g., male, female, boy, girl, man, woman, etc.). Level 2 words had sex, age, and marriage-kinship features (e.g., brother, sister, husband, wife, bride, and groom). Level 3 words contained sex, age, marriagekinship, and royalty-religion features (e.g., king, queen, prince, princess, priest, and nun). Access probabilities and CRPs were predicted to increase the higher the level, since each successive level has more features than the previous level. When access probabilities and CRPs

Table 3

Mean Access Probabilities and Conditional Recall Probabilities (CRPs) for Five Categories of Nouns

\begin{tabular}{lcccccc}
\hline & \multicolumn{4}{c}{ Noun Category } \\
\cline { 2 - 6 } & $\begin{array}{c}\text { Ab- } \\
\text { stract }\end{array}$ & $\begin{array}{c}\text { Con- } \\
\text { crete }\end{array}$ & Male & Female & Sex \\
\hline Access Probability & .33 & .67 & .56 & .54 & .46 \\
CRP & .65 & .87 & .80 & .79 & .69 \\
\hline
\end{tabular}


were computed for each level of male and female nouns, they showed the predicted trend. CRPs were $.66, .80$, and .89 for Levels 1,2, and 3, respectively. This trend was statistically significant when item variance was used as error $\left[F_{2}(2,41)=8.30, p<.05\right]$. The access probabilities for Levels 1,2 , and 3 were also significantly different $\left[.32, .54\right.$, and $.66, \min \mathrm{F}^{\prime}(2,41)=8.91$, $\mathrm{p}<.05]$.

\section{Discussion}

It has long been known in memory research that sentence recall protocols are fragmentary. According to the holistic chunking model, fragmentary recalls are products of two types of errors. The first type of error is attributed to trace decoding. When a sentence trace is accessed, some of the propositions in a trace may not be recovered. When subjects fail to recover a proposition, they either delete the elements of that proposition or they attempt to fill in what is missing by guessing words or phrases. These types of errors generate intrusions that are unrelated to the presented items.

A second type of recall error occurs when there is a failure in pattern-match processes during word reconstruction. Whenever a proposition is retrieved, the subjects select lexical items that capture the recovered attributes of each proposition element. Sometimes the correct word is reconstructed, sometimes a semantically or phonologically similar word is reconstructed (intrusion), and sometimes subjects are unable to find a word that sufficiently captures the configuration of available attributes (omissions). The intrusions that are generated from these pattern-match errors are always related conceptually to the presented words.

The pattern of subject- and object-noun intrusions supported the notion that noun elements encoded within a proposition virtually always include certain general semantic features. Only $.031(14 / 452)$ of the subject- and object-noun intrusions were inconsistent with the semantic categories of the presented nouns (see Table 2), that is, abstract, concrete, male, and female. These features are invariantly encoded when propositions are acquired, at least for the class of sentences examined. It is plausible that certain lexical features are very salient and automatically incorporated within the semantic interpretation of a proposition in a data-driven fashion (Hayes-Roth \& Hayes-Roth, 1977). It is important to emphasize that these features cannot be directly inferred or reconstructed from information about the other words in the sentences. In particular, the sentences were carefully prepared so that the co-occurrence restrictions of the verbs would not completely predict the semantic categories of the subject, object, and argument nouns.

If patterns of unrelated intrusions are used as criteria for determining the propositional structure of sentences, at least some of the sentences in this study contained two propositions: PROP1 (verb, subject, object) and PROP2 (proposition, PROP1, argument). The hypothesis that subject and object arguments are directly related to transitive verbs has been supported in some studies that have used memory or reaction time data for testing theories of representation (Hayes-Roth, 1977; Moore, 1972). There is other behavioral evidence that additional sentence arguments are not directly related to transitive verbs, particularly when these arguments refer to time and location information (Dosher, 1976; Kintsch, 1974).

It is premature to specify how other classes of sentences are chunked into propositions at acquisition. Weinreich (1963) has proposed that there is an upper limit of three arguments per proposition and that the limit is a language universal. This limit falls within the alleged nodal limitations of attention, immediate memory, long-term memory, and consciousness (Estes, 1972; Graesser \& Mandler, 1978; Mandler, 1967; Miller, 1956). It is plausible that the number of elements would need to fall within the limited span of apprehension in order for elements in a proposition to be integrated holistically.

It was argued earlier that patterns of intrusion errors provide more appropriate tests of the trace structure of sentences than do access probabilities and CRPs. However, access probabilities and CRPs are good indices of the success of pattern-match processes that are assumed to operate during trace access and word reconstruction. The success of pattern-match processes varied to a large extent among different noun categories. These variations were systematic. Pattern matches were most successful when nouns had many features in their lexical representations. The more complex nouns (with many features) are more distinctive and less confusable with alternative lexical items. These variations among different noun categories will ultimately obscure tests for sentence structure, particularly when investigators allow biases in sampling items for different sentence positions (cf. Clark, 1965). The range in access probabilities and CRPs among different noun categories was 7 to 11 times the range among different sentence positions.

Whereas access probabilities and word reconstruction probabilities may not provide defensible tests of the trace structure of sentences, the holistic chunking model can provide an impressive simulation of these data. The assumptions of the holistic chunking model have been incorporated in a mathematical model that attempts to account for the recall proportions of specific combinations of words (Graesser, 1977). ${ }^{1}$ Given that there are 4 possible prompt words in a sentence, and 8 possible combinations of recall (or nonrecall) of the three words to be recalled, there are $32(4 \times 8)$ recall combinations altogether. Although it is impossible to claim that the mathematical model provided a perfect fit to the recall proportions, there were no serious deviations between 
the obtained and expected recall proportions. In fact, there was a nonsignificant chi square when obtained and expected proportions were compared.

\section{EXPERIMENT 2}

Experiment 1 supported the holistic chunking model's prediction that intrusion errors for subject and object nouns would always be related to presented nouns. Experiment 2 was designed to push this strong prediction to its limits. The acquisition sentences in Experiment 2 were transitive sentences that sometimes contained nouns that violated the verb's co-occurrence restrictions, for example, "the tray loved the house." The verb "love" requires an animate subject noun, yet the sentence contains an inanimate noun. The intrusions for noun violations should always preserve the lexical features of the presented nouns if (1) certain lexical features ( \pm abstract and \pm animate) are invariantly incorporated in the proposition trace and (2) the course of trace reconstruction preserves the nouns' encoded attributes instead of feature constraints imposed by the verb's co-occurrence restrictions. According to the holistic chunking model, the intrusions for "tray" should include inanimate nouns (e.g., cup, desk, radio, etc.), but not animate nouns (e.g., man, aunt, person, etc.), even though the animate nouns agree with the verb's co-occurrence restrictions.

It is quite conceivable that the intrusion errors for noun violations would drift toward noun categories that agree with the verb's co-occurrence restrictions (e.g., tray $\rightarrow$ person). There are three ways in which noun intrusions would be unrelated to presented nouns. First, subjects might not encode the lexical feature -animate when the feature violates the verb's co-occurrence restrictions. If this occurs, then it would be incorrect to claim that certain lexical features are invariantly incorporated in a proposition trace. Second, the lexical feature -animate might be incorporated in the proposition trace during acquisition, but reconstructive processes might transform the feature to tanimate, which is consistent with the verb's cooccurrence restrictions. Third, subjects might not encode any attributes for nouns that violate the verb's cooccurrence restrictions, and during word reconstruction they would guess a noun with features that agree with the verb. If any one of the above three processes occurs, then some proportion of the intrusion errors will be unrelated to presented nouns when the presented nouns violate the verb's co-occurrence restrictions.

\section{Method}

Subjects. Sixty undergraduate students at California State University, Fullerton, participated in this experiment as a psychology course requirement.

Materials. Acquisition sentences were divided into nine blocks of six sentences. Each sentence had a subject-verb-object format. with transitive verbs presented in the past tense. The nouns were preceded by the article "the" (e.g., the bachelor disliked the barn).
Two classes of verbs were selected when generating the acquisition sentences. Half of the verbs required animate subject nouns, whereas any noun category could occur in the object position (e.g., disliked, wanted, forgot, etc.). The other half of the verbs required animate object nouns, and any category of noun could occur in the subject position (e.g., upset, threatened, interested, etc.).

The subject and object nouns were sampled equally from three categories: abstract, inanimate concrete, and animate. The nouns were randomly assigned to the subject and object positions of each verb class; the two classes of verbs had an equal distribution of abstract, concrete, and animate nouns in both sentence positions. This procedure of assigning nouns to sentences produced anomalous sentences that violated the verb's co-occurrence restrictions. Two sentences in each block were subject-violation sentences, in which abstract or concrete nouns were assigned to verbs that required animate subject nouns (e.g., the hope studied the grandfather, and the tray loved the house). Two sentences in each block were object-violation sentences, with abstract or concrete nouns assigned to verbs that required animate object nouns (e.g., the baby surprised the premise, and the inference bored the wall). The other two sentences in each block were normal sentences. The normal, subject-violation, and object-violation sentences were randomly ordered within each block, and two sets of materials were constructed with different random orderings.

Procedure. The subjects were instructed to study sets of sentences for the purpose of recalling them in a memory test. The subjects were told that some sentences were "weird, metaphorical, or difficult to conceptualize." They were encouraged to study each sentence as well as they could, regardless of how difficult it was to understand its meaning. During acquisition of each block of six sentences, an experimenter read aloud each sentence, allowing $10 \mathrm{sec}$ for study per sentence. Immediately after the last sentence was presented, the experimenter read aloud the verbs, one at a time; the subjects wrote down the verb plus the subject and object nouns that occurred with the verbs. The subjects were allowed $20 \mathrm{sec}$ for recall for each verb prompt. The verb prompts were presented in the same order as their corresponding acquisition sentences were presented. Consequently, there was a 1 - to $2-$ min delay between the acquisition and recall of any given sentence.

\section{Results}

Table 4 shows the proportion of noun intrusions that were scored as unrelated to the nouns presented

Table 4

Intrusion Data and Measures of Correct Recall for Subject and Object Nouns in Normal, Subject-Violation (SV), and Object-Violation (OV) Sentences: Experiment 2

\begin{tabular}{|c|c|c|c|c|}
\hline & \multirow[b]{2}{*}{ Sentence } & \multicolumn{3}{|c|}{ Sentence Category } \\
\hline & & Normal & SV & ov \\
\hline & \multicolumn{4}{|c|}{ Access Probability } \\
\hline \multirow[t]{2}{*}{$\mathrm{p}(\mathrm{S}$ or $\mathrm{O}$ or $\mathrm{SO})$} & & .63 & .47 & .55 \\
\hline & \multicolumn{4}{|c|}{ Conditional Recall Probability } \\
\hline \multirow[t]{2}{*}{$\begin{array}{l}\mathrm{p}(\mathrm{S} / \mathrm{O}) \\
\mathrm{p}(\mathrm{O} / \mathrm{S})\end{array}$} & $\begin{array}{l}\text { Subject } \\
\text { Object }\end{array}$ & $\begin{array}{l}.87 \\
.85\end{array}$ & $\begin{array}{l}.79 \\
.84\end{array}$ & $\begin{array}{l}.89 \\
.78\end{array}$ \\
\hline & \multicolumn{4}{|c|}{ Conditional Intrusion Probability } \\
\hline$\frac{\text { Intrusion }}{\text { Intrusion + Omission }}$ & $\begin{array}{l}\text { Subject } \\
\text { Object }\end{array}$ & $\begin{array}{l}.52 \\
.50\end{array}$ & $\begin{array}{l}.21 \\
.49\end{array}$ & $\begin{array}{l}.47 \\
.18\end{array}$ \\
\hline Intrusion + Omission & \multicolumn{4}{|c|}{$\begin{array}{c}\text { Probability of Emitting an } \\
\text { Unrelated Intrusion }\end{array}$} \\
\hline Unrelated Intrusion & Subject & .000 & .048 & .000 \\
\hline All Intrusions & Object & .000 & .033 & .030 \\
\hline
\end{tabular}


in the acquisition sentences. A noun intrusion was scored as unrelated if it was not in the same category (abstract vs. concrete vs. animate) as the presented noun. Separate proportions are reported for subject and object nouns in the three sentence categories: normal, subject-violation, and object-violation. Just as in Experiment 1 , an incorrectly recalled noun was scored as an intrusion only when the other noun in the sentence was recalled correctly; this constraint served to insure that the correct sentence trace was recovered. Table 4 also includes three other measures of mer.ıry performance: (1) access probabilities, that is, the proportion of observations in which one or two nouns were recalled correctly, (2) CRPs, that is, the probability of recalling a noun correctly given that the other noun in the sentence was recalled correctly, and (3) a conditional intrusion probability, that is, the probability of emitting an intrusion given that the recall protocol involved either an intrusion or an omission.

The primary purpose of Experiment 2 was to examine whether subjects generated unrelated intrusions in sentence positions that violated the verb's co-occurrence restrictions. Table 4 shows, and statistical analyses confirm, that the proportion of unrelated intrusions approached zero in all sentence positions and sentence categories. There were 54 noun intrusions from sentence positions that violated the verb's co-occurrence restrictions and only 2 of these were categorically unrelated to the presented nouns. Of those sentence positions that were consistent with the verb's co-occurrence restrictions, only 2 out of 208 intrusions were categorically unrelated to the presented noun. These data indicate that errors of reconstruction preserve the features of the presented nouns even when the features violate linguistic demands of the sentence verb.

Additional analyses revealed that subjects were reluctant to guess when nouns were incongruent with the verb's co-occurrence restrictions. When a presented noun violated the verb constraints and subjects were unable to reconstruct a noun that captured its trace attributes, subjects tended to omit a response altogether. This conclusion is based on the pattern of scores that computed the probability of emitting an intrusion, given that a protocol contained either an intrusion or an omission: $p$ (intrusion/intrusion or omission). These conditional intrusion probabilities were significantly lower in the two cells that involved noun violations (.20) than in the four cells that did not involve noun violations $(.50)\left[F_{2}(1,102)=19.72, p<.05\right]$.

Not surprisingly, the pattern of access probabilities and CRPs indicated that it was easier to access and integrate words in normal sentences than in sentences with subject violations or object violations. The mean access probability was significantly greater for normal sentences (.63) than for sentences with either subject violations or object violations (.51) $\left[\min F^{\prime}(1,168)=5.84, p<.05\right]$. The mean CRP for nouns that violated the verb's co-occurrence restrictions was significantly lower than the mean CRP for nouns that were congruent with the verb constraints $[.79$ vs. .86 , respectively, $\left.\min \mathrm{F}^{\prime}(1,182)=6.58, \mathrm{p}<.05\right]$.

\section{Discussion}

The analysis of noun intrusions in Experiment 2 supported both the holistic chunking model and the hypothesis that certain lexcial features are invariantly incorporated in proposition traces at acquisition. The noun intrusions virtually always contained the same lexical features, \pm abstract and tanimate, as the presented nouns. The noun intrusions preserved these features even when the presented nouns violated the co-occurrence restrictions of the verb. The fact that the proportion of unrelated noun intrusions approached zero (.019) supports the holistic chunking model's assumption that the SVO proposition is encoded holistically and retrieved in an all-or-none manner.

The results of Experiment 2 revealed an additional property of word reconstruction processes during sentence recall. The reconstruction of one word in a recovered proposition may be inhibited when available attributes are inconsistent with constraints imposed by other words in the proposition. The incidence of both correct recalls and intrusion errors was attenuated when a noun violated the co-occurrence restriction of the verb. However, the recovered attributes of noun elements were not transformed or deleted by the constraints imposed by other words, at least with regard to rather distinctive lexical features (i.e., \pm abstract and \pm animate).

\section{GENERAL DISCUSSION}

The intrusion analyses reported in Experiments 1 and 2 supported the holistic chunking model of sentence acquisition and retrieval. According to this model, sentences are segmented into proposition units during acquisition and these units are holistic encodings. When a proposition is recovered at retrieval, there are some attributes available for each element within a proposition. However, access to one proposition does not insure access to other propositions in a sentence. The functional holistic units are apparently not always entire sentences. Instead, propositions are encoded holistically and retrieved as units. Consequently, further support accumulates for propositional theories of sentence representation (Clark \& Clark, 1977; Kintsch, 1974). Although the analyses of noun intrusions uniformly supported the holistic chunking model, it should be acknowledged that the tests of holism and propositional segmentation heavily rest on the data from the final prepositional phrase in the SVOA sentences. The holistic chunking model clearly needs to be tested further with other sentence frames.

A few colleagues have expressed mild dissatisfaction with the present tests for sentence structure, which are based primarily on patterns of intrusion errors. The source of this dissatisfaction resides in the fact that intrusions occur in a relatively small proportion (approximately .10) of the recall protocols. This fact is, perhaps, unfortunate but does not, and should not, 
undermine the holistic chunking model and the issues that this study has addressed. The holistic chunking model does provide an impressively good fit to patterns of correct recall (Graesser, 1977), in addition to patterns of intrusions. But, more importantly, analyses of intrusions provide more appropriate tests of the model than do quantitative patterns of correct recall. It has been argued throughout this study that patterns of correct recall reveal little or nothing about sentence structure per se. Instead, patterns of correct recall are more critically sensitive to pattern-match processes inherent in trace access and word reconstruction.

This study has examined sentence acquisition and retrieval in a rather unnatural setting. The sentences were unrelated to each other and were divorced from a referential context that would normally be shared between speakers and listeners. Subjects were found to group words into propositions and the segmentation was guided by semantic properties of verbs. It is plausible that the functional units are different when sentences are embedded in connected discourse (Carpenter \& Just, 1976; Fodor, 1976; Fodor et al., 1974; Haviland \& Clark, 1974; Miller \& Johnson-Laird, 1976; Norman et al., 1975). The holistic chunking model could, in principle, be expanded to accommodate predictable variations in sentence structure when more is understood about processes at supersentential levels. Structural variations could again be tested by observing patterns of intrusion errors.

\section{REFERENCES}

ANDERSon, J. R. Language, memory, and thought. Hillsdale, N.J: Erlbaum, 1976.

ANDERson, J. R., \& Bower, G. H. On an associative trace for sentence memory. Joumal of Verbal Learning and Verbal Behavior, 1971, 10, 672-680.

ANDERson, J. R., \& Bower, G. H. Human associative memory. Washington, D.C: Winston, 1973.

ANDERSON, R. C. Substance recall of sentences. Quarterly Journai of Experimental Psychology, 1974, 26, 530-541.

Anderson, R. C., \& Ortony, A. On putting apples into bottles: A problem of polysemy. Cognitive Psychology, 1975, 7, 162-180.

Asch, S. E. A reformulation of the problem of associations. American Psychologist, 1969, 24, 92-102.

Blumenthal, A. L. Prompted recall of sentences. Journal of Verbal Learning and Verbal Behavior, 1967, 6, 203-206.

Blumenthal, A. L., \& Boakes, R. Prompted recall of sentences: A further study. Journal of Verbal Learning and Verbal Behavior, 1967, 6, 674-676.

Brewer, W. F. Memory for ideas: Synonym substitution. Memory \& Cognition. 1975, 3, 458-464.

Carpenter, P. A., \& Just, M. A. Models of sentence verification and linguistic comprehension. Psychological Review, 1976, 83, 318-322.

Сномsкy, N. Aspects of the theory of syntax. Cambridge, Mass: M.I.T. Press, 1965.

CLARK, H. H. Some structural properties of simple active and passive sentences. Journal of Verbal Learning and Verbal Behavior. 1965, 4. 365-370.

Clark, H. H. The language-as-a-fixed-effect fallacy: A critique of language ritatistics in psychological research. Journal of Verbal
Learning and Verbal Behavior, 1973, 12, 335-359.

Clark, H. H., \& Clark, E. V. Psychology and language: An introduction to psycholinguistics. New York: Harcourt-Brace, 1977.

Dosher, B. A. The retrieval of sentences from memory: A speed-accuracy study. Cognitive Psychology, 1976, 8, 291-310.

Estes, W. K. An associative basis for coding and organization in memory. In A. W. Melton \& E. Martin (Eds.), Coding processes in human memory. Washington, D.C: Winston, 1972.

Fillmore, C. J. The case for case. In E. Bach \& R. T. Harms (Eds.), Universals in linguistic theory. New York: Holt, Rinehart, \& Winston, 1968.

Fodor, J. A. The language of thought. New York: Thomas Crowell, 1976.

Fodor, J. A., Bever, T. G., \& 'GARRETT, M. F. The psychology of language. New York: McGraw-Hill, 1974.

Foss, D. J., \& HARwood, D. A. Memory for sentences: Implications for human associative memory. Journal of Verbal Learning and Verbal Behavior, 1975, 14, 1-16.

GrAESSER, A. C. Sentence memory and comprehension. Unpublished doctoral dissertation, University of California, San Diego, 1977.

Graesser, A. C., \& Mandler, G. Limited processing constrains the storage of unrelated sets of words and retrieval from natural categories. Journal of Experimental Psychology: Human Learning and Memory, 1978,4, 86-100.

GREEN, D. W. The effects of tasks on the representation of sentences. Journal of Verbal Learning and Verbal Behavior. 1975, 14, 275-283.

GREENo, J. G. How associations are memorized. In D. A. Norman (Ed.), Models of human memory. New York: Academic Press, 1970.

Haviland, S. E., \& Clark, H. H. What's new? Acquiring new information as a process in comprehension. Journal of Verbal Learming and Verbal Behavior, 1974, 13, 512-521.

HAYES-Roth, B. Evolution of cognitive structures and processes. Psychological Review, 1977, 84, 260-278.

Hayes-RoTh, B., \& Hayes-Roth, F. The prominence of lexical information in memory representations of meaning. Journal of Verbal Learning and Verbal Behavior, 1977, 16, 119-136.

Horowitz, L. M., \& Prytulak, L. S. Redintegrative memory. Psychological Review, 1969, 76, 519-531.

JENKINS, J. J. Remember that old theory of memory? Well, forget it! American Psychologist, 1974, 29, 785-795.

Johnson, N. H. Organization and the concept of a memory code. In A. W. Melton \& E. Martin (Eds.), Coding processes in human memory. New York: Winston, 1972.

Kahneman, D. Attention and effort. Englewood Cliffs, N.J: Prentice-Hall, 1973.

Katz, J. J., \& Postal, P. M. An integrated theory of linguistic descriptions. Cambridge, Mass: M.I.T. Press, 1974.

KINTSCH, W. The representation of meaning in memory. Hillsdale, N.J: Erlbaum, 1974.

Kosslyn, S. M., \& Pomerantz, J. R. Imagery, propositions, and the form of internal representations. Cognitive Psychology, $1977,9,52.76$.

Kučera, H., \& Francis, W. W. Computational analysis of present-day American English. Providence, R.I: Brown University Press, 1967.

Mandler, G. Organization and memory. In K. W. Spence \& J. T. Spence (Eds.). The psychology of learning and motivation (Vol. 1). New York: Academic Press, 1967.

Marschark, M., \& Paivio, A. Integrative processing of concrete and abstract sentences. Joumal of Verbal Learning and Verbal Behavior, 1977, 16, 217.231.

Miller, G. A. The magical number seven, plus or minus two: Some limits on our capacity for processing information. Psychological Ra'view'. 1956. 63, 81-97. 
MilleR, G. A. A psychological method to investigate verbal concepts. Journal of Mathematical Psychology, 1969, 6, 169-191.

Miller, G. A., \& Johnson-LaIRd, P. N. Language and perception. Cambridge, Mass: Harvard University Press, 1976.

Moore, T. E. Speeded recognition of ungrammaticality. Journal of Verbal Learning and Verbal Behavior, 1972, 11, 550-560.

Murbock, B. B. Human memory: Theory and data. New York: Wiley, 1974.

Norman, D. A., \& Bobrow, D. G. On data limited and resource limited processes. Cognitive Psychology, 1975, 7, 44-64.

Norman, D. A., Rumelhart, D. E., \& The LNR Research Group. Explorations in cognition. San Francisco: Freeman, 1975.

Rumelhart, D. E., \& Ortony, A. The representation of knowledge in memory. In R. C. Anderson, R. J. Spiro, \& W. E. Montague (Eds.), Schooling and the acquisition of knowledge. Hillsdale, N.J: Erlbaum, 1976.

Simmons, R. Semantic networks: Their computation and use for understanding English sentences. In R. C. Schank \& $K$. $M$. Colby [Eds.], Computer models of thought and language. San Francisco: Freeman, 1973.
SInger, M. Thematic structure and the integration of linguistic information. Journal of Verbal Learning and Verbal Behavior, 1976, 15, 549-558.

ThORNDyke, P. S. Conceptual complexity and imagery in comprehension and memory. Journal of Verbal Learning and Verbal Behavior, 1975, 14, 259-369.

Tulving, E. Ecphoric processes in recall and recognition. In J. Brown (Ed.), Recall and recognition. London: Wiley, 1976. WANNER, H. E. On remembering, forgetting, and understanding sentences: $A$ study of the deep structure hypothesis. Unpublished dissertation, Harvard University, 1968.

WEINREICH, U. On the semantic structure of language. In J. H. Greenberg (Ed.), Universals of language. Cambridge, Mass: M.I.T. Press, 1963.

\section{NOTE}

1. These data and analyses can be obtained by writing the author.

(Accepted for publication August 7,1978 .) 